\title{
Comparison of SBR process and DAT-IAT process and advantages and drawbacks of both processes
}

\author{
Zixuan Liu \\ (Harbin Institute of Technology, School of Municipal and Environmental Engineering Harbin \\ Heilongjiang Province, China, 150090)
}

zx liu1226@outlook.com

Keywords: DAT-IAT; SBR; Removal of Phosphorus

Abstract:SBR process is similarwith DAT-IAT process on operating procedures, the biggest difference is whether the process is able to accept the continuous influent or not, the advantages of SBR process is that the operation and equipment is simple and quality of effluent is good. In terms of DAT-IAT process, the advantages include low investment, strong impact resistance but the biggest drawback is the poor effect of phosphorus removal due to the short anaerobic duration and lack of carbon.

\section{Introduction}

Sequencing batch activated sludge treatment system, referred to as SBR process, is a biological wastewater treatment technology. Withrapid development of computer and automatic control technology, complex problems of the initial development of intermittent operationcan be solved, so the advantages of the process can be fully developed.

Led by the Chinese engineer Xiuduo Wang, several engineers invented an improved type of SBR process--DAT-IAT process, its reaction mechanism and pollutant removal mechanisms is basically the same with traditional SBR process, only the structure and running operations are different. The advantages of the process consist of land saving, high stability and low investment. The process has shown a strong vitality and broad prospects, is being proved by the practice in recent years.

\section{Comparison of operations in SBR process and DAT-IAT process}

The operation mode of DAT-IAT is the same with the typical SBR reactor as its operation consists of five phases: influent, reaction, sedimentation, drainageand idle, but the specific requirements of its operations are different with SBR method, the elaboration of the five stages is followed:

1. Influent phase

Unlike typical SBR process, the raw water continuously flows into the DAT, and then enters the IAT. Continuous influent makes the influent control greatly simplified, such a dual pool system also avoids hydraulic short circuit.

2. Reaction phase

Varying from the different biochemical reactions occurring in the same reaction pool in SBR process, the reaction phase of DAT-IAT divides into two parts: There is continuous aeration in the DAT pool, the water is in a completely mixed flow state in the pool, where most of the organic matter is to be degraded; the mixture treated by DAT enters continuously into IAT through a diversion system between the two pools, intermittent aeration of IAT further removes organics, making the effluent meet the discharge standards.

3. Precipitation phase

Precipitation occurs directly after stopping aeration or stirring in the reaction pool in SBR process, while in terms of DAT-IAT process, precipitation occurs only in the IAT, the activated sludge floc is basically at a static state and the solid-liquid separation is occurring at the same time when the aeration is stopped in the IAT pool. The flow rate of the mixture is very low from DAT to IAT, making no disturbance to the IAT, thus the precipitation of IAT is generally more efficient than dynamic precipitation of secondary settling tank. 


\section{Drainage phase}

As for SBR process, drainage phase also occurs in the reaction pool, while drainage of DAT-IAT process occurs only in IAT, when the water level rises to the highest level of the pool, the precipitation phase ends, the supernatant was slowly discharged with the start of the decanter at the end of the IAT pool, and the drainage stops when the water level drops to the lowest level.

5. Idle phase

After the completion of IAT drainage phase, a running cycle ends, the intermittent time period between two cycles is idle phase. For SBR reactor, the length of time of the process or whether to cancel the process can be determined by the quality of the sewage and treatment requirements. For DAT-IAT process, the continuous water results in no need for a standby system.

Both operations of the two processes are much the same, but with different specific requirements,a clear comparison can be seen in Table 1.

Table 1. DAT-IAT and SBR basic situation and performance comparison

\begin{tabular}{c|c|c}
\hline Process Type & Conventional SBR & DAT-IAT \\
\hline Items & Rectangular Pool & DAT-IAT Series \\
\hline Influent Form & Intermittent & Continuous \\
\hline Drainage Form & Intermittent & Intermittent \\
\hline Aeration & Intermittent & $\begin{array}{c}\text { DAT Continuous } \\
\text { IAT Intermittent }\end{array}$ \\
\hline Precipitation & Static State & Semi-Static State \\
\hline Cycle (h) & $4 \sim 8$ & 3 or 4 \\
\hline Volume Utilization & $50 \sim 70$ & Yes \\
\hline Sludge Return & No & Good \\
\hline Prevent Sludge Bulking & Above Average & Above Average \\
\hline Nitrogen Removal & Good & Fair \\
\hline Phosphorus Removal & Good & Treatment Plants \\
\hline Practicability & Small Sewage & Treatment Plants \\
\hline
\end{tabular}

\section{Advantages and drawbacks}

\subsection{SBR process}

The most important feature of SBR process is that the flowing state is completely mixed in the aeration pool, and in terms of organic degradation, it is actually a chronological plug flow, the matrix content of organic matter is degraded as time extends. The biggest advantage of the SBR process is to run aeration, reaction, sedimentation, drainage and other unit operations repeatedly in a chronological order in one reaction pool. A typical SBR process can be divided into five stages by running order, called respectively influent phase, reaction phase, precipitation phase, drainage phase, and idle phase, the time required for the five phases is called a cycle. As the treatment process of numerous sewage treatment plants, there are several advantages about SBR process: (1) the equipment is simple; (2) the operation is simple; (3) good quality of treated water; (4) good effects of nitrogen and phosphorus removal.

However, SBR method has some limitations and unavoidable defects due to its own design: (1) a 
single SBR reactor requires a larger pool when there is influx of continuous water; (2) for multiple SBR reactor, the valve of its water and drainage should be automatically switched frequently; (3)SBR reactor can not achieve the continuous feeding of sewage; (4) a high vacancy rate of the equipment; (5) a large loss of sewage lifting head.

\subsection{DAT-IAT process}

The most important feature of DAT-IAT is that the process is divided into two parts, raw sewage first gets preliminary biological treatment in DAT pool and then into the IAT pool, the continuous aeration plays a role in water balance, improves the stability of the entire process, the influx process occurs only in the DAT pool. DAT pool is the main reaction zone, also known as aerobic tank, there is continuous inflow of sewage including mixture returning from IAT into the reaction zone, to be continuous aerated, giving full play to the activated sludge biological degradation, and most of the soluble organic contamination is removed. DAT can be seen as a flexible, comprehensive pre-reactor, so that the DAT and IAT pool pool can maintain longer SRT and high concentration of MLSS, and have a strong impact resistance to organic load and poisons. Drainage process occurs only in the IAT pool, so this allows the adjustability of the entire biological treatment system to further enhance and facilitate removal of organic matter. IAT is equivalent to a conventional SBR pool, but the inflow of water is continuous, aeration is cyclical, the treated sludge supernatant and excess sludge are completed within the IAT. Because of the functions of DAT to adjust the water quality and balance the quantity, sothe quality of water is stable, the organic load is low and adaptability to changes in water quality is also improved in IAT pool. Also C / $\mathrm{N}$ is low in IAT pool which is conducive to breeding bacteria nitrification, making the nitrification occur. Because the intermittent aeration can form continued alternating environment of aerobic - anoxic - anaerobic, it can get some nitrogen and phosphorus removal at the same time with the removal of $\mathrm{BOD}_{5}$.

Although DAT-IAT process has some advantages like low infrastructure investment, high capacity utilization, simple water system, a strong resistance to poison and organics etc., the process has its corresponding disadvantages: (1) a large amount of return sludge results in a high energy consumption; (2) necessary to extend the operation cyclein order to remove nitrogen and phosphorus; (3) a poor result of removal of phosphorus.

The poor removal of phosphorus is the biggest drawback of DAT-IAT process, in practical engineering operation, the wastewater treatment plant with this processgenerally uses dosage to remove phosphorus, spends a large amount of money on the dosage. Because the process is of a non-limiting aeration process type, meaning the aeration is at the same with influent, unlike conventional SBR process, anaerobic conditions can be maintained in the influent phase by stirring in order to promote the full release of phosphorus of phosphorus removal bacteria. On the one hand, anaerobic process occurs only in the late phaseof drain in DAT-IAT phase, the duration is so short that phosphorus can not be fully released and the release of phosphorus is influencedbadly by the residual oxygen and concentration of $\mathrm{NOx}-\mathrm{N}$ in the IAT pool. On the other hand, DAT is a continuous aeration pool, removes most of the dissolved organic pollutants. When the sewage flowsinto the IAT pool, easily degradable organic matter is almost exhausted, the value of BOD / TKN is very low, with insufficient carbon, denitrifying bacteria can only use a slow and complex biological degradation of organic compounds as carbon source or by endogenous respiration reaction, denitrification rate is very slow; and anaerobic time is short,the state changing from aerobic to anaerobic will take some time, these factors were not conducive to denitrification, resulting in the high concentration of $\mathrm{NO}_{3}-\mathrm{N}$, this in turn affects the release of phosphorus of phosphorus removal bacteria, which leads in the decreased capacity of uptake of phosphorus in the next cycle of aerobic state, so the removal of phosphorus is greatly reduced in the system. Thus, while DAT-IAT process has certain capacityof phosphorus removal, but the content of phosphorus can not meet the emission standards.

\section{Conclusions}

As an improved process of SBR, DAT-IAT process is approximately the same with SBR process in operation, both processes consist of 5 phases: influent, reaction, sedimentation, drainage and idle, 
but the specific requirements for DAT-IAT process is different as the process is divided into two parts, continuous aeration in DAT pool, intermittent operation in IAT pool, which can make the influent continuous.

As for SBR process, the equipment and operation is simple and the quality of effluent is good, but it can not achieve the continuous feeding of sewage and the cycle is long while the water level change is great. It is suitable for small sewage treatment plants according to these features. With the advantages of small infrastructure investment, high capacity utilization and a strong resistance to poison and organics, DAT-IAT process also has its drawbacks: a high energy consumption because of large amounts of return sludgeand poor effects of phosphorus removal due to the short anaerobic duration and lack of carbon. However, it still applies to larger sewage treatment plants.

\section{References}

[1]YunlongYang,QibinChen: Status Quo and Development of the Sequencing Batch Reactor in Industrial Water \& Wastewater, Vol.33, No.2, 2002, In Chinese

[2]Pu Chen,HuafengHu: Nitrogen and Phosphorus by Modified DAT-IAT Technology in Environmental Science \& Technology, Vol.36, No.2, Feb.2013, In Chinese

[3]Daqun Zhang, XiuduoWang: New Process of SBR—DAT-IAT and New Decanter, Beijing, 1996, In Chinese

[4]Xia Zhu, Guangsheng Fan: Experimental Study on Treatment of Urban Sewage by DAT-IAT Technology in Research Progress, Apr. 2007, In Chinese

[5]Irvine R.L., Busch A.W.: Sequencing Batch Biological Reactors-An Overview. JWPCF, 51:235, 1979 